\title{
841 TARGETING THE INTERSECTION OF IMMUNOLOGY AND REDOX BIOLOGY: THERAPEUTIC POTENTIAL OF AN ANTI-THIOREDOXIN ANTIBODY
}

Gabriella Willman, Max Davidson, Carolina Trkulja, Owe Orwar, Sachin Bhat*. Oblique Therapeutics AB, Göteborg, Sweden

Background Human Thioredoxin 1 ( $\operatorname{Tr} x 1)$ was initially discovered as Adult $\mathrm{T}$ cell Leukemia Derived Factor (ADF) that is capable of inducing IL-2R/Tac (CD25) in T and NK cells. ${ }^{1}$ Serum Trx1 levels have been correlated to incidence and severity of disease in melanoma patients ${ }^{2}$ and elevated serum Trx1 levels have been reported in hepatocellular carcinoma ${ }^{3}$ and pancreatic cancer. ${ }^{4}$ In contrast to extracellular Trx1, the role of intracellular Trx1 in redox biology is extensively studied (reviewed in 5). The role of $\operatorname{Trx} 1$ as a cytokine in regulating immune cells is less well explored. In order to understand the role of Trx1 in cancer immunology and evaluate the potential of anti-Trx1 monoclonal antibody $(\mathrm{mAb})$ as a therapeutic, anti-Trx1 mAbs were generated and evaluated.

Methods mAbs against full length human Trx1 were generated in mice using standard immunization protocol. The antibodies were tested for their binding affinity to human Trx1 and cross-reactivity with mouse Trx1 using sandwich ELISA method. mAbs were screened for their ability to neutralize hTrx1 in a functional assay where naïve human CD4+ cells were polarized to differentiate into $\mathrm{T}$ regulatory cells (Tregs). Effects of the mAbs in vivo were tested in a mouse syngeneic tumor model as well as a tumor model in humanized mice.

Results Five unique mAbs were extensively characterized in in vitro and in vivo models. All five mAbs bound hTrx1 with high affinity with an EC50 of $<1 \mathrm{nM}$ in a sandwich ELISA assay. To develop a screening assay for anti-Trx mAbs, the ability of exogenously added Trx1 to increase the level of expression of CD25 on human naïve peripheral CD4+ cells activated with anti-CD3 and anti-CD28 antibodies and polarized with TGFb1 and IL-2 was assessed. Trx1 increases the level of expression of CD25 on CD $4+\mathrm{CD} 25+$ cells as well as CD $4+C D 25+F O X P 3+$ cells. In this Treg polarization assay, four of the five anti-Trx mAbs showed a statistically highly significant effect in reducing the $\%$ CD $4+\mathrm{CD} 25+$ as well as $\mathrm{CD} 4+\mathrm{CD} 25+\mathrm{FOXP} 3+$ cells within viable cells. In addition, there was a dramatic reduction in the expression levels of CD25 and FOXP3 in the respective cell populations. The ability of these antibodies to modulate immune cells within the tumor microenvironment and have an anti-tumor effect in a syngeneic model (using a mouse cross-reactive antibody) and a humanized mouse tumor model was investigated. Results from these studies will be presented.

Conclusions We describe a novel immunomodulator pathway in tumor immunology.

Acknowledgements We acknowledge Proteogenix for production of Trx mAbs, Aquila Biomedical for screening Trx mAbs in Treg polarization assay and Champions Oncology for running tumor models.

\section{REFERENCES}

1. Yutaka et al. (1989). EMBO J 8(3):757-765.

2. Wang et al. (2015). Oncolmmunology 4(9):e1027471.

3. Miyazake et al. (1998). Biotherapy 11:277-288.

4. Nakamura et al. (2000). Cancer Detect Prev 24:53-60.

5. Collet JF, Messens J. (2010). Antioxidants \& Redox Signaling 13(8):1205-1216.

6. Mougiakakos et al. (2011). Blood 17(3):857-861.

http://dx.doi.org/10.1136/jitc-2021-SITC2021.841 